\section{CONFERENCE REPORT}

\section{British Orthodontic Society Training Grade Group Meeting}

'So you want to become an orthodontist' was held at the Hilton Double Tree Hotel in Leeds on 8 November 2013. This conference was organised by Chris Barker and Donnchadh O'Marain of the Training Grades Group of the British Orthodontic Society (BOS) with over 50 delegates in attendance from various career backgrounds, notably Dental Foundation Trainees, senior house officers and general dental practitioners who were all keen to find out more about a career in orthodontics as well as gaining an insight into the National Orthodontic Recruitment Process.

I chose to travel to the conference from Glasgow after hearing excellent reviews from several colleagues who had applied for Orthodontic Training Programmes the previous year and who had found the one-day conference an excellent first step in planning for their future applications. 2014 will be my first application and this was an ideal way for me to gather my thoughts and plan for the forthcoming round of interviews.

The conference was slick, well organised and presented by speakers who were consultants and registrars working in various NHS trusts with a wealth of experience in the Orthodontic National Recruitment Programme.

Topics covered on the day met delegates' demands. Simon Littlewood spoke about 'The StR orthodontic programme and research in orthodontics' and also about BOS, followed by Paul Cook speaking on 'National Recruitment, applying for orthodontics and the orthodontic interview'. David Waring gave helpful tips on how to build your $\mathrm{CV}$ and get into orthodontics and Ian Edwards spoke about the role of a consultant orthodontist. It is always useful to find out exactly what specialist training is like and this was portrayed in an excellent manner

by two trainees from

Leeds, Sophy Barber and Katherine Forde. Finally, Guy Deeming gave a superb lecture on 'Life in a specialist practice'.

My favourite part of the day was the informal group discussion session which allowed small groups of attendees to discuss the interview process with an assigned orthodontic registrar. It also gave delegates an insight into the demands of the Orthodontic StR training programme, the research component of the programme and the various Master degrees that are run.

Delegates were given an opportunity to quiz the registrars about their experience of the interview process, Do's and Don'ts as well as how to prepare for application, the portfolio and the interview stations. This was a fitting way to end an all-round excellent event.

In summary, this conference was a great success and reinforces BOS' activities and commitment to its young members pursuing a career in orthodontics. The conference is held twice yearly in Leeds and London respectively and is an event not to be missed by anyone who is wishing to pursue a career in orthodontics, especially for those applying for National Recruitment in March 2014.

BY AMMAR AL HOURANI, SHO IN RESTORATIVE DENTISTRY AND ORAL SURGERY

\title{
WINNING COVERS IMPRESS WELSH GOVERNMENT
}

The popular 'children's drawings' series of BDJ covers (Volume 215, JulyDecember 2013) are to feature in a public display in the Senedd - the Welsh government building - between 22 January and 14 February.

The covers feature the 12 winning entries to a competition for schoolchildren in Wales called 'Designed to Smile'

\section{BOOK REVIEWS}

\section{MUCOGINGIVAL ESTHETIC SURGERY}

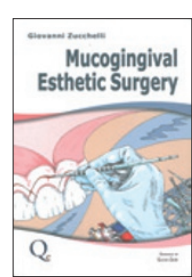

G. Zucchelli (ed)

Quintessence

price $£ 240.00$; pp 830

ISBN 9788874921713

This exquisitely illustrated, extensive book explains the art and science of aesthetic surgical techniques on the gingivae around implants and natural teeth. The main aim of the book is the treatment of lesions caused by gingival recession and to augment gingival thickness and height around prosthesis or implants, with the goal of satisfying the patient's aesthetic concerns.

The author has extensive experience in periodontal plastic surgery and has received many awards for his clinical research into periodontology in the USA and Europe.

Diagnosis, pathogenesis and prognosis of mucogingival defects are discussed at great length along with excellent diagrams and clinical photographs. Various surgical techniques are comprehensively covered, including sections on treatment protocols, flap designs, suturing techniques, surgical equipment and post-operative care. Again, the use of clinical photography is exemplary at demonstrating surgical techniques.

All in all, this is a great book for every periodontist and implantologist. Budding oral surgeons will also find this book useful for the sections on flap design and suturing techniques. The book is suitable for the beginner as well as the more experienced clinician. Do not be overwhelmed by the sheer size of this book, as this is due to the many excellent photographs and diagrams! The step by step procedures, world class photography and the skills of the author make this book pleasure to read.

P. GILL

\section{SMOKING BANS BOOST QUIT ATTEMPTS}

Completely banning tobacco use inside the home - or more broadly in the whole city - measurably boosts the odds of smokers either cutting back or quitting entirely.

Researchers from California - the first state in the world to ban smoking in public places in 1994 - surveyed 1,718 smokers and found that total home smoking bans were significantly associated with quit attempts in males, but not females. ${ }^{1}$ Total home bans were more effective in households without children, possibly reflecting the ultimate goal of cessation rather than primarily reducing children's second-hand smoke exposure.

The researchers said: 'Having both home and city bans on smoking appears to be even more effective'.

1. Zablocki R W, Edland S D, Myers M G et al. Smoking ban policies and their influence on smoking behaviors among current California smokers: a population-based study. Prev Med 2013; DOI: 10.1016/j. ypmed.2013.11.018 